\section{Comparison of two methods of surface profile extraction from multiple ultrasonic range measurements}

To cite this article: Billur Barshan et al 2000 Meas. Sci. Technol. 11833

View the article online for updates and enhancements.

\section{Related content}

\author{
Fast processing techniques for accurate \\ ultrasonic range measurements \\ Billur Barshan \\ Robust ultrasonic range finder-an FFT \\ analysis \\ P Holmberg \\ - Improved range estimation using simple \\ infrared sensors \\ Çari Yüzbaiolu and Billur Barshan
}

\section{Recent citations}

- Virtual source aperture imaging with auto-
focusing of unknown complex geometry
through dual layered media
Ewan Hoyle et al
- Measurement of the cavitation pattern by
$\underline{\text { two non-intrusive techniques: laser }}$
imaging and ultrasound pulsed
Alberto Felici et al
Experimental and Numerical Investigation
of Liquid Slosh Behavior Using Ground-
Based Platforms
Ran Zhou et al

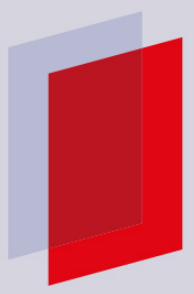

\section{IOP ebooks'}

Bringing you innovative digital publishing with leading voices to create your essential collection of books in STEM research. Start exploring the collection - download the first chapter of every title for free. 


\title{
Comparison of two methods of surface profile extraction from multiple ultrasonic range measurements
}

\author{
Billur Barshan and Deniz Başkent $\dagger$ \\ Department of Electrical Engineering, Bilkent University, Bilkent, TR-06533 Ankara, Turkey \\ E-mail: billur@ee.bilkent.edu.tr
}

Received 21 October 1999, in final form 4 February 2000, accepted for publication 4 April 2000

\begin{abstract}
Two novel methods for surface profile extraction based on multiple ultrasonic range measurements are described and compared. One of the methods employs morphological processing techniques, whereas the other employs a spatial voting scheme followed by simple thresholding. Morphological processing exploits neighbouring relationships between the pixels of the generated arc map. On the other hand, spatial voting relies on the number of votes accumulated in each pixel and ignores neighbouring relationships. Both approaches are extremely flexible and robust, in addition to being simple and straightforward. They can deal with arbitrary numbers and configurations of sensors as well as synthetic arrays. The methods have the intrinsic ability to suppress spurious readings, crosstalk and higher-order reflections, and process multiple reflections informatively. The performances of the two methods are compared on various examples involving both simulated and experimental data. The morphological processing method outperforms the spatial voting method in most cases with errors reduced by up to $80 \%$. The effect of varying the measurement noise and surface roughness is also considered. Morphological processing is observed to be superior to spatial voting under these conditions as well.
\end{abstract}

Keywords: ultrasonic ranging systems, sonar, range measurement, morphological processing, voting, feature extraction, surface profile extraction, map building

\section{Introduction}

An inexpensive, yet effective and reliable approach to machine perception is to employ multiple simple range sensors coupled with suitable data processing. The two approaches described in this paper, morphological processing and a spatial voting scheme followed by thresholding, are applied to ultrasonic range measurements to reconstruct the profiles of arbitrarily curved surfaces that are encountered in unstructured environments such as mines, rough terrain or underwater. In contrast, approaches based on geometrical or analytical modeling are often limited to elementary target types with constant or piecewise-constant curvature $[1,2]$. The methods presented here are novel ways of processing range data in the form of an arc map which represents angular uncertainties. These methods can also be viewed as new ways of solving a class of nonlinear reconstruction problems that arise when a large number of sensors produce range measurements. These nonlinear inverse problems do not seem amenable to efficient solution by standard analytical or

$\dagger$ Current address: Department of Biomedical Engineering, University of Southern California, Los Angeles, CA 90089-1451, USA. numerical techniques. Both methods presented are extremely flexible and can easily handle arbitrary sensor configurations as well as synthetic arrays obtained by moving a relatively small number of sensors. In contrast, approaches based on geometrical or analytical modelling have often been limited to simple, well structured sensor configurations.

A commonly noted disadvantage of ultrasonic ranging systems is the difficulty associated with handling spurious readings, crosstalk, higher-order and multiple reflections. The proposed methods are capable of effectively suppressing the first three of these, and have the intrinsic ability to make use of echo returns beyond the first one (i.e. multiple reflections) so that echoes returning from surface features further away than the nearest can also be processed informatively.

It is important to underline that morphological processing and spatial voting are employed here to process the ultrasonic arc map of the surface being reconstructed, rather than conventional camera images.

The methods presented in this paper, which are based on the use of multiple range sensors combined with arc map processing, can be applied to different physical modalities of 
range sensing of vastly different scales and in many different areas. These may include radar, sonar, optical sensing and metrology, remote sensing, ocean surface exploration, geophysical exploration, robotics and acoustic microscopy. Although the present paper deals with the determination of two-dimensional surface profiles, both methods can be readily generalized to three-dimensional environments [3].

This paper is organized as follows. In section 2, basic principles of ultrasonic ranging systems are reviewed. In section 3, the generation of arc maps is described and two different methods are introduced for processing these arc maps. Section 3.1 presents a scheme based on morphological processing. Section 3.2 describes a spatial voting scheme followed by simple thresholding. The methods are compared based on various examples in section 3.4 based on both simulated and experimental data. The effect of additive noise on the measurements and the effect of surface roughness are considered in sections 3.7 and 3.8 respectively.

\section{Basics of ultrasonic ranging systems}

Although the methods presented in this paper can be applied to other kinds of range sensor or measurement, here we concentrate on ultrasonic range sensing from which our experimental results are derived. We consider simple ultrasonic transducers that measure time-of-flight (TOF) $t_{0}$, which is the round-trip travel time of the pulse between the transducer and the surface. Given the speed of transmission $c$, the range $r$ can be easily calculated from $r=c t_{0} / 2$. Many ultrasonic transducers operate in this pulse-echo mode [4]. The same transducer can function as both the receiver and the transmitter. The major limitation of ultrasonic transducers comes from their large beamwidth. For example, the Polaroid transducers used in this study have a half beamwidth angle of $\theta_{0}= \pm 12.5^{\circ}$. Although these devices return accurate range data, usually they cannot provide direct information on the angular position of the surface from which the reflection is obtained. Thus, all that is known is that the reflection point lies on a circular arc whose radius is determined by $r=c t_{0} / 2$, as illustrated in figure 1(a). More generally, when one sensor transmits and another receives, it is known that the reflection point lies on the arc of an ellipse whose focal points are the transmitting and receiving elements (figure $1(\mathrm{~b})$ ). Notice that the arcs are tangent to the reflecting surface at the actual point(s) of reflection. The angular extent of these arcs is determined by the sensitivity regions of the transducers. For the same transmitting/receiving pair, if multiple echoes are detected at the receiver, circular or elliptical arcs are drawn to correspond to each echo. As a result of this process, we obtain the arc map, comprised of circular and elliptical arcs. An example of an arc map is given in figure 2(a).

We distinguish between multiple reflections and higherorder reflections as follows. Multiple reflections are those which are caused by one time (or first-order) reflection of the emitted wave from different points in the environment, resulting in multiple returns at the detector. For instance, along the curved surface, there may be multiple points falling within the sensitivity region of the transducer at which the incoming beam is perpendicular to the surface tangent. In general, such points will be at different distances from the transducer, creating echoes at locations corresponding to these distances. Such multiple reflections have been modelled in the simulations and also properly registered by the experimental detection circuitry so that the reflections are processed informatively. Some simpler systems detect and process only the first echo received, corresponding to the nearest reflection point.

A higher-order reflection refers to a single return detected after bouncing off successively from more than one point in the environment before returning to the detector. The key idea of the method is that a large number of data points coincide with the actual surface (at least at the tangent points of the arcs) and the data points off the actual curve are more sparse. Those spurious arcs caused by higher-order reflections and crosstalk also remain sparse and lack reinforcement most of the time. The algorithms eliminate these spurious arcs together with the sparse arc segments resulting from the angular uncertainty of the sensors. Although higher-order reflections have not been generated in the simulated data sets due to the complexity of modelling them, they naturally exist in the experimental data, providing the opportunity to verify the ability of our method to eliminate them.

We initially consider the case where the surfaces reflect the ultrasonic waves specularly (mirror-like). Since most airborne ultrasonic systems operate at resonance frequencies below $200 \mathrm{kHz}$, the propagating waves have wavelengths well above several millimetres. Thus, the features on the objects which are smaller than the wavelength cannot be resolved, resulting in specular reflections [2]. Later, we also consider rough surfaces that do not reflect the waves specularly. The ultrasonic devices modelled in the simulation studies and used in the experiments are Polaroid 6500 series transducers [5], operating at a resonance frequency $f_{0}=49.4 \mathrm{kHz}$, which corresponds to a wavelength of $\lambda=c / f_{0}=6.9 \mathrm{~mm}$ at room temperature.

Most commonly, the large beamwidth of the transducer is accepted as a device limitation that determines the angular resolving power of the system and the reflection point is assumed to be along the line of sight. In this naive approach, a range reading of $r$ from a transmitting/receiving transducer is taken to imply that an object lies along the line of sight of the transducer at the measured range. Consequently, the angular resolution of the surface profile measurement is limited by the rather large beamwidth, which is a major disadvantage. Our approach, as will be seen, turns this disadvantage into an advantage. Instead of restricting oneself to an angular resolution equal to the beamwidth by representing the reflection point as a coarse sample along the line of sight, circular or elliptical arcs representing the uncertainty of the object location are drawn. By combining the information inherent in a large number of such arcs, angular resolution far exceeding the beamwidth of the transducer is obtained.

\section{Processing of the arc map}

As an illustrative example, figure 2(a) shows the arc map obtained from a surface using an irregular sensor configuration. A considerably large number of arcs can be obtained with a reasonable number of sensors because each sensor can receive pulses transmitted from all the others, 


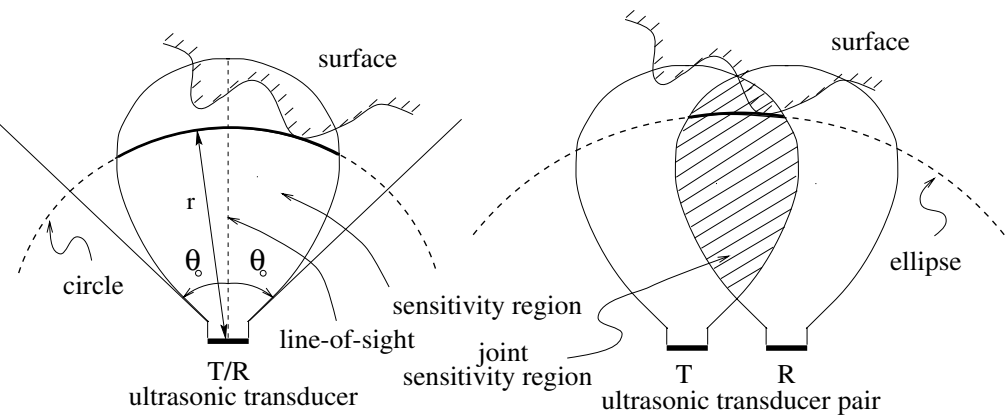

(a)

(b)

Figure 1. (a) For the same transducer transmitting and receiving, the reflecting point is known to be on the circular arc shown. (b) The elliptical arc if the wave is transmitted and received by different transducers. The intersection of the individual sensitivity regions serves as a reasonable approximation to the joint sensitivity region.

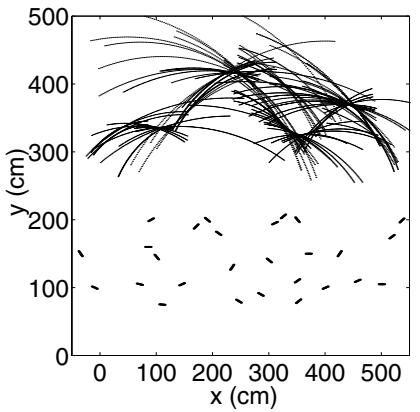

(a)

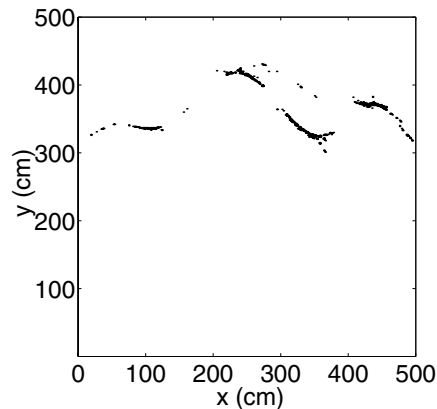

(b)

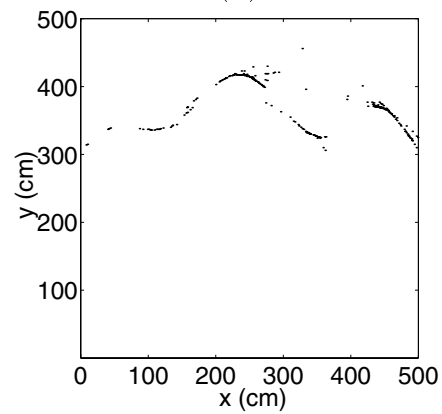

(d)

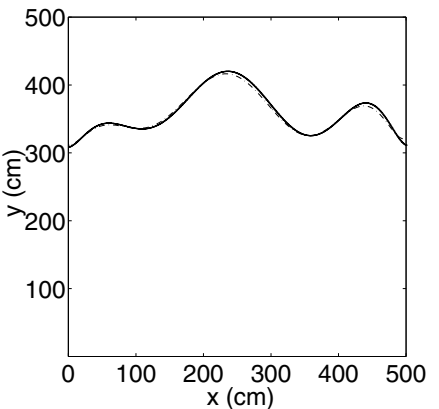

(c)

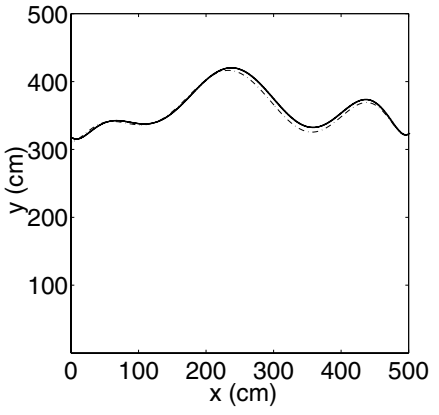

(e)

Figure 2. (a) The arc map obtained with an array of 27 sensors, each of $45^{\circ}$ beamwidth. Features visible between about $y=100 \mathrm{~cm}$ and $y=200 \mathrm{~cm}$ are representations of the transducers. (b) The result of $n=6$ thinning, (c) the fitted curve of order $m=8$ (solid line) and the original surface (dashed line). $E_{1}=2.85 \mathrm{~cm}, E_{2}=0.098$. (d) The result of spatial voting and subsequent thresholding. (e) Polynomial fit of order $m=8$ to part (d) (solid line), and the actual surface (dashed line). $E_{1}=4.42 \mathrm{~cm}, E_{2}=0.152$ (simulated data).

provided a reflection point lies in the joint sensitivity region of that sensor pair. For sensors with large beamwidth, the number of arcs obtained approaches the square of the number of sensors. Near the actual reflection point(s), several arcs intersect with small angles. The many small segments of the arcs superimposed in this manner tend to coincide with and cover the actual surface, creating the darker features in figure 2(a) that reveal the surface profile. The remaining parts of the arcs, not actually corresponding to any reflections and simply representing the angular uncertainty of the sensors, remain more sparse and isolated. Similarly, those arcs caused by higher-order reflections, crosstalk and noise also remain sparse and lack reinforcement.

It is often possible to visually perceive the surface profile from the darker features in the arc map (see for example figures 4, 5 and 7). The purpose of the methods described in this paper is to formalize and automate this process. The dark features of the arc maps which eventually reveal the surface profile are essentially caused by two effects: several arc segments slightly displaced with respect to each other, and several arcs crossing at the same pixel. The first of the two methods discussed below exploits the neighbouring relationship of the arc segments whereas the second method takes into account the number of arcs intersecting at each pixel.

\subsection{Morphological processing}

As a first alternative to processing the arc map, morphological processing is considered. In this approach, morphological 
operators are used to weed out the sparse and isolated arc segments, spikes or extrusions in the arc map, leaving behind the mutually reinforcing segments that directly reveal the original surface profile.

Erosion, dilation, opening, closing and thinning are widely used morphological operations to accomplish tasks such as edge detection, skeletonization, segmentation, texture analysis, enhancement and noise removal in image processing [6]. Mathematical morphology has been applied in diverse areas such as pattern and shape analysis [7], machine vision [8], medical imaging [9], remote sensing [10], automatic target recognition [11], flaw detection [12] and electron microscopy [13]. Although most applications involve processing of conventional binary or grey-scale images, in some cases, range images are processed where the range information is coded in the grey levels of the image [14-16]. The present approach is novel in that morphological processing is applied to range measurements in the form of an arc map, representing angular uncertainties.

Morphological operations basically consist of a set of simple rules to modify images. Erosion and dilation are two fundamental morphological operations used to thin and fatten an image respectively. A simple algorithm for erosion is as follows: if all eight neighbours of a pixel with value one also equal one, that pixel preserves its value, otherwise its value is set equal to zero. This operation erodes or shrinks the image in all directions by one pixel. An example is presented in figure 3. On the other hand, the dilation operation is used to fatten an image. This time, all eight neighbours of those image pixels which originally equal one are set equal to one.

Thinning is a generalization of erosion with a parameter $n$ varying in the range $1 \leqslant n \leqslant 8$. In this case, it is sufficient for any $n$ of the neighbours of an image pixel to equal one in order for that pixel to preserve its value of one. This parameter is useful for adjusting the amount of thinning so as to obtain the best results.

In pruning, which is a special case of thinning, at least one $(n=1)$ of the neighbouring pixels must have the value one in order for the central pixel to remain equal to one after the operation. This operation is used to eliminate isolated points [6]. As should be evident, pruning and erosion are the two extremes of thinning, corresponding to $n=1$ and $n=8$ respectively.

In some cases, the direct use of erosion may eliminate too many points and result in the loss of information characterizing the surface. For such cases, the compound operations of opening and closing are considered. Opening consists of erosion followed by dilation, and vice versa for closing. Opening helps reduce small extrusions, whereas closing enables one to fill the small holes inside the image [17]. Closing is applied prior to thinning in cases where the points are not closely connected to each other so that the direct use of thinning may result in the loss of too many points. Filling the gaps using closing first may prevent this from happening.

The result of applying $n=6$ thinning to the arc map shown in figure 2(a) is presented in figure 2(b). Note that the points remaining after morphological processing follow the surface profile quite closely.

\subsection{Spatial voting}

Another way of processing the information in the arc map is to employ a spatial voting scheme followed by thresholding. In this approach, the number of arcs which cross at each pixel is kept track of while generating the arc map. A matrix is created which represents the number of arcs crossing at each pixel. The values of pixels which have not been crossed by any arcs remain zero. The values of other pixels are equal to the number of arcs crossing them. Note that the number of arcs crossing a given pixel was not directly employed in the morphological processing approach. The morphological processing method makes use of only the binary information as to whether a pixel has been crossed by at least one arc or not. On the other hand, the spatial voting scheme takes into account how many arcs cross at a given pixel.

The most important parameter in the voting method is the threshold value. A suitable threshold level is chosen to select those pixels which have been crossed more frequently. If the number of crossings for a given pixel is less than the threshold, the value of that pixel is set equal to zero. If the number is greater than or equal to the threshold, the pixel value is set equal to one.

As an example, consider again the arc map illustrated in figure 2(a). The result obtained with spatial voting and subsequent thresholding is given in part $(d)$ of the same figure.

\subsection{Curve fitting}

Both methods generate a set of points which outline the surface. To represent this estimated surface profile compactly, we obtain a least-squares polynomial fit to these points. Since the error distribution after morphological processing is zero mean and symmetric, application of a leastsquares fit is appropriate. The curve fitted to the result of morphological thinning given in figure 2(b) is displayed in figure 2(c). Similarly, the curve fitted to the result of spatial voting and thresholding in figure 2(e) is displayed in part (f) of the same figure. Two error measures, both comparing the final polynomial fit with the actual curve, are introduced:

$$
\begin{gathered}
E_{1}=\sqrt{\frac{1}{N} \sum_{i=1}^{N}\left[p\left(x_{i}\right)-y\left(x_{i}\right)\right]^{2}} \\
E_{2}=\frac{E_{1}}{\sigma_{y}} .
\end{gathered}
$$

The first is a root-mean-square absolute error measure, whereas the second is a dimensionless relative error measure with respect to the variation of the actual curve. $N$ is the total number of columns in the map matrix, $p\left(x_{i}\right)$ are the samples of the fitted polynomial and $\sigma_{y}^{2}=\frac{1}{N} \sum_{i=1}^{N}\left[y\left(x_{i}\right)\right.$ $\left.-\frac{1}{N} \sum_{i} y\left(x_{i}\right)\right]^{2}$ is the variance of the actual surface profile $y\left(x_{i}\right)$.

In the example given in figure 2 , the errors obtained with the first method are $E_{1}=2.85 \mathrm{~cm}$ and $E_{2}=0.098$. On the other hand, the errors obtained with the second method are $E_{1}=4.42 \mathrm{~cm}$ and $E_{2}=0.152$, which are larger than those obtained with the first method by a factor of 1.55 . 


\begin{tabular}{|l|l|l|l|l|l|}
\hline 1 & 1 & 1 & 1 & 1 & 0 \\
\hline 1 & 1 & 1 & 1 & 1 & 0 \\
\hline 1 & 1 & 1 & 1 & 1 & 1 \\
\hline 0 & 1 & 1 & 1 & 0 & 0 \\
\hline
\end{tabular}

(a)

\begin{tabular}{|l|l|l|l|l|l|}
\hline 0 & 0 & 0 & 0 & 0 & 0 \\
\hline 0 & 1 & 1 & 1 & 0 & 0 \\
\hline 0 & 0 & 1 & 0 & 0 & 0 \\
\hline 0 & 0 & 0 & 0 & 0 & 0 \\
\hline
\end{tabular}

(b)

Figure 3. An example for erosion: (a) the original image, (b) and the image after erosion.

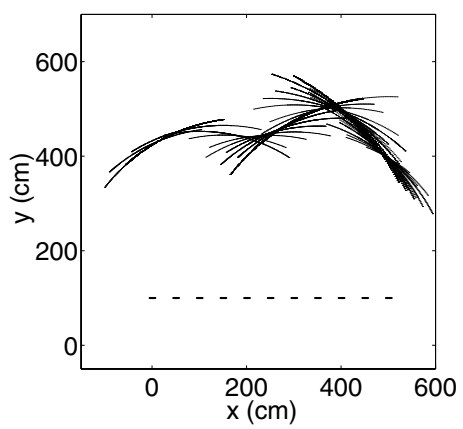

(a)

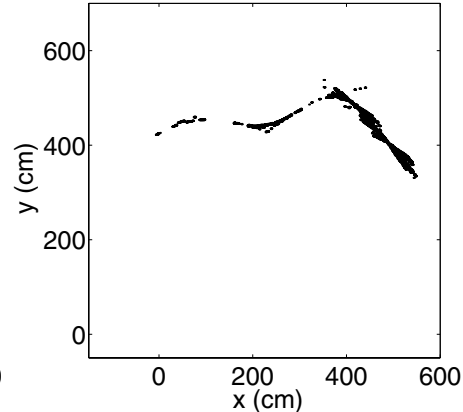

(b)

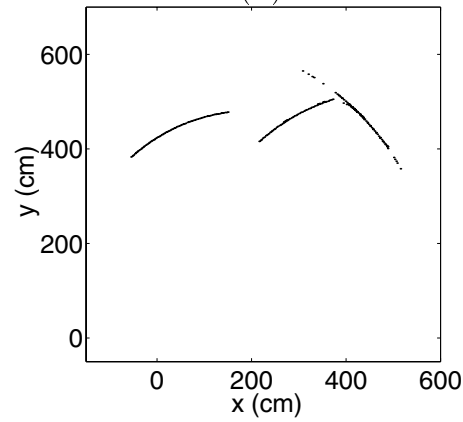

(d)

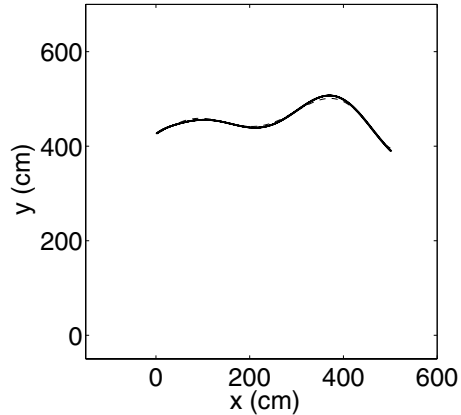

(c)

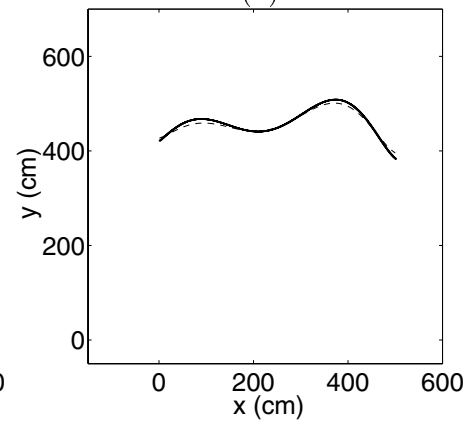

(e)

Figure 4. (a) The arc map obtained from a linear array of 11 sensors with $50 \mathrm{~cm}$ spacing where the sensors are individually rotated from $40^{\circ}$ to $140^{\circ}$ in $10^{\circ}$ steps. (b) Result of closing and $n=5$ thinning applied to part (a). (c) Polynomial fit of order $m=8$ to part (b) (solid line), compared with the actual surface (dashed line). $E_{1}=3.03 \mathrm{~cm}, E_{2}=0.127$. (d) The result of spatial voting and subsequent thresholding. (e) Polynomial fit of order $m=7$ to part (d) (solid line), and the actual surface (dashed line). $E_{1}=5.42 \mathrm{~cm}, E_{2}=0.227$ (simulated data).

\subsection{Comparison of the two methods}

In this section, we illustrate and compare the two approaches with various examples involving different numbers and configurations of ultrasonic range sensors.

First, we consider a linear array of sensors. This array has an horizontal extension of $5.0 \mathrm{~m}$ with $50 \mathrm{~cm}$ spacings between the 11 sensors, as shown in figure 4 . Given the narrow beamwidth of the sensors $\left(25^{\circ}\right)$, the number of arcs obtained with this many sensors turns out to be insufficient to reconstruct the surface profile [18]. Whereas narrow beamwidths are esteemed for their higher resolving power in conventional usage of ultrasonic transducers, here it would have been desirable to have sensors with larger beamwidths. This would have enabled a greater number of the sensor pairs at hand to produce elliptical arcs, better revealing the surface profile. Instead, we have considered the alternative strategy of rotating the sensors and refiring them several times in order to collect a sufficient number of arcs.

A further consideration is that in practice the number of sensors may be limited. One way to overcome this limitation is to move a smaller array much in the same spirit as synthetic aperture radar (SAR) techniques [19]. However, this is not exactly equivalent to the full array since those elliptical arcs corresponding to pairs of sensors not contained within the actually existing array will be missing.

We now return to figure 4(a) where the sensors are individually rotated around their shown positions. The result of morphological processing is presented in part (b), and the polynomial fitted is shown in part (c) of the same figure, with $E_{1}=3.03 \mathrm{~cm}$ and $E_{2}=0.127$. Corresponding results for spatial voting are given in parts (d) and (e) of the figure, with $E_{1}=5.42 \mathrm{~cm}$ and $E_{2}=0.227$, which is 1.79 times larger.

In the second example, five transducers located on a circular arc have been used, where the array is moved over a distance of $2.0 \mathrm{~m}$ to collect data. The circular configuration corresponds to the arrangement of ultrasonic sensors on the Nomad 200 mobile robot [20] in our laboratory. The resulting arc map is shown in figure 5(a). The result of $n=6$ thinning and curve fitting are shown in parts (b) and (c) of the same figure respectively, with errors $E_{1}=1.64 \mathrm{~cm}, E_{2}=0.200$. 


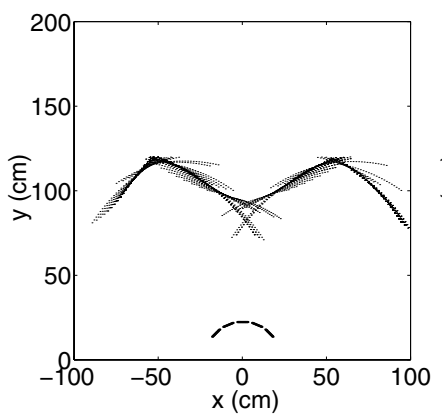

(a)

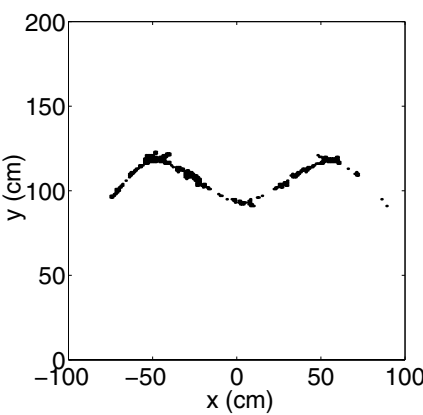

(b)

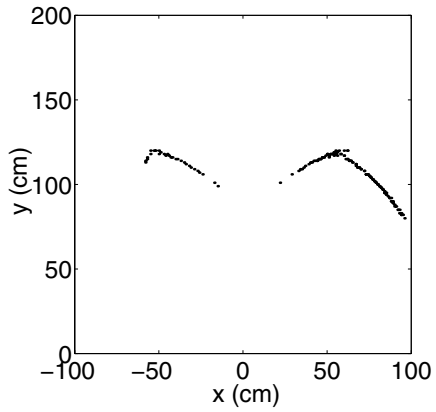

(d)

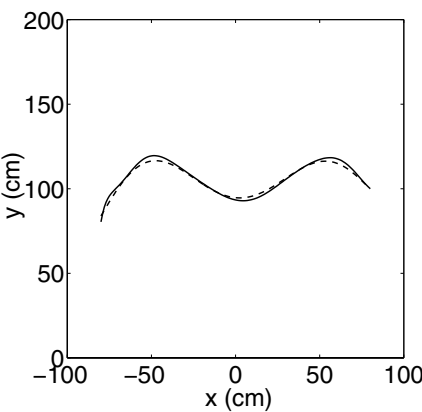

(c)

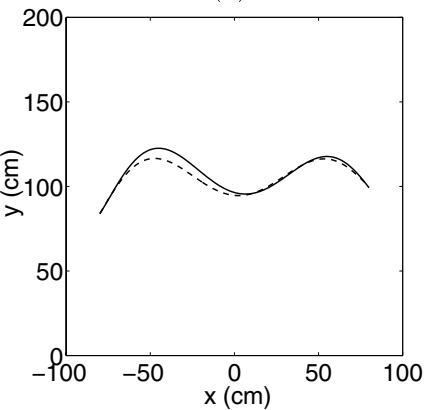

(e)

Figure 5. (a) The arc map obtained by translating the circular array of sensors from $(-100,0)$ to $(100,0)$, collecting data every $5 \mathrm{~cm}$. (b) The result of $n=7$ thinning applied to part (a). (c) Polynomial of order $m=12$ fitted to part (a) (solid line) compared with the original surface (dashed line). $E_{1}=1.64 \mathrm{~cm}, E_{2}=0.200$. (d) The result of spatial voting and subsequent thresholding. (e) Polynomial fit of order $m=6$ to part (d) (solid line), and the actual surface (dashed line). $E_{1}=3.35 \mathrm{~cm}, E_{2}=0.410$ (simulated data).

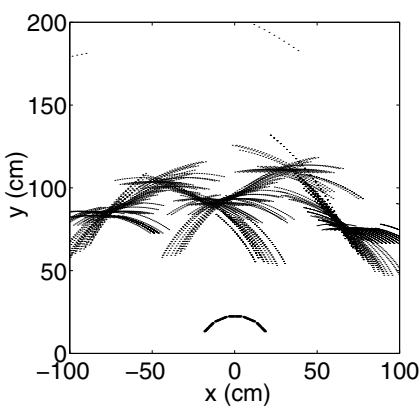

(a)

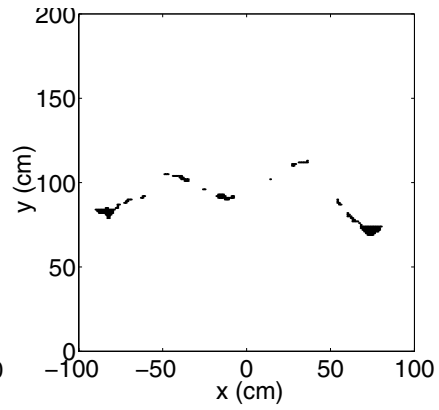

(b)

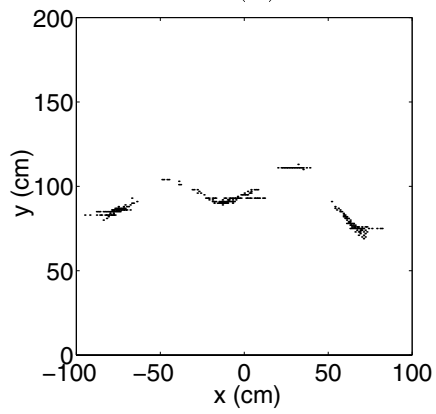

(d)

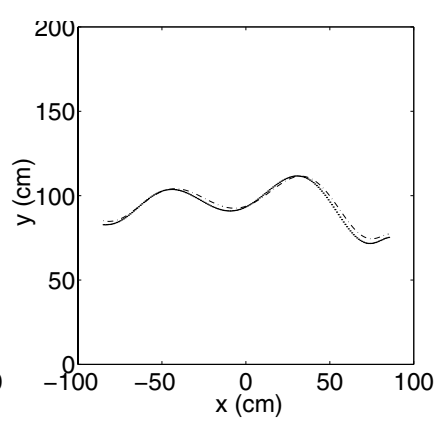

(c)

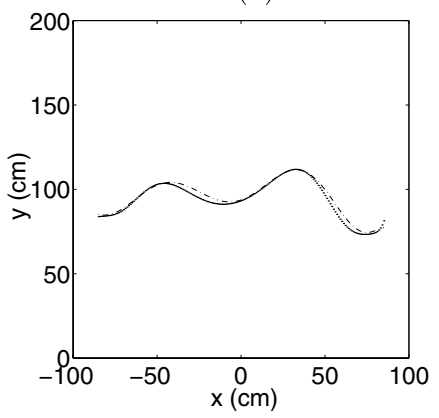

(e)

Figure 6. (a) The arc map and the sensor configuration. The data are collected from the surface at every $2.5 \mathrm{~cm}$ by translating the array from $(-75,0)$ to $(75,0)$. (b) Result of erosion and $n=7$ thinning applied to part (a). (c) Polynomial fit of order $m=8$ to part (b) (solid line), compared with the actual surface (dashed line). $E_{1}=2.09 \mathrm{~cm}, E_{2}=0.204$, (d) The result of spatial voting and subsequent thresholding. (e) Polynomial fit of order $m=12$ to part (d), resulting in $E_{1}=2.12 \mathrm{~cm}, E_{2}=0.206$ (experimental data).

The result of spatial voting and subsequent thresholding is shown in part (d). In this case, the errors are $E_{1}=3.35 \mathrm{~cm}$, $E_{2}=0.410$, which is more than twice the error obtained with morphological processing.
As another example, consider the experimentally obtained arc map shown in figure 6(a). These data were collected with a real ultrasonic ranging system, from a cardboard surface constructed in our laboratory. An array 


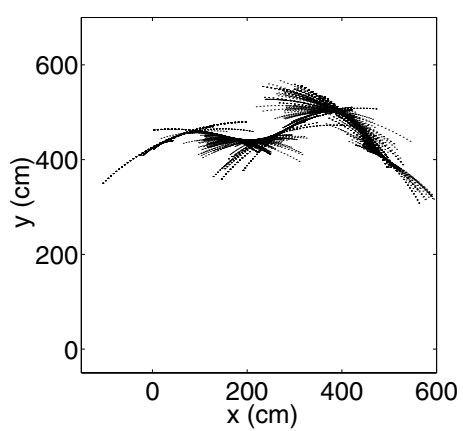

(a)

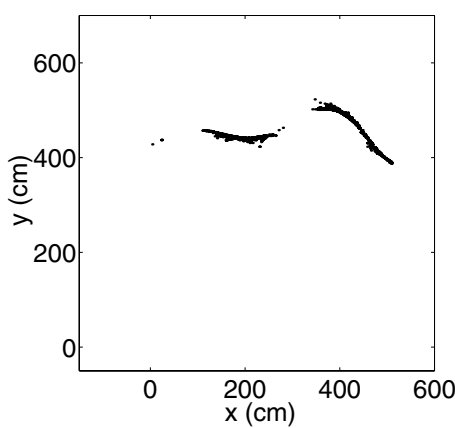

(b)

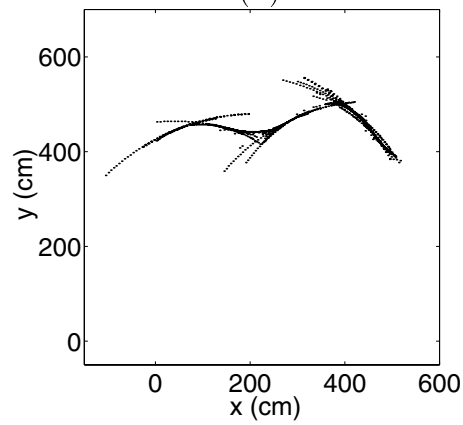

(d)

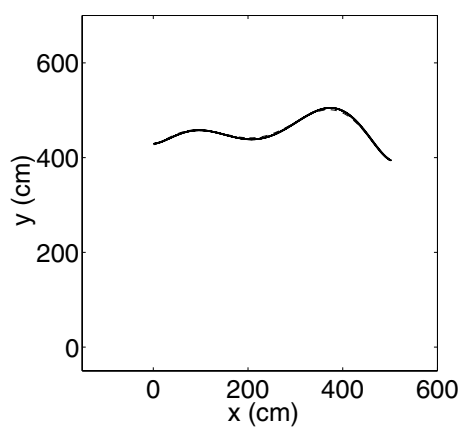

(c)

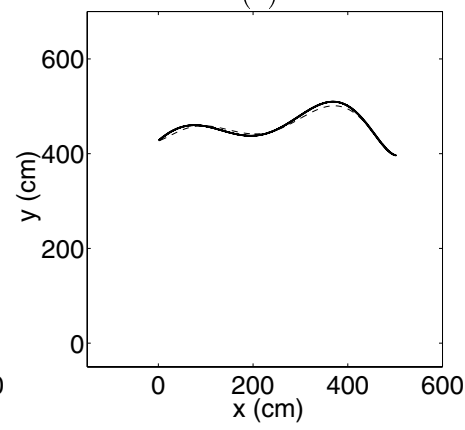

(e)

Figure 7. (a) The arc map obtained by using 150 sensors positioned and oriented randomly (not shown). The $x$ and $y$ coordinates of each sensor are independent and uniformly distributed in the intervals $[0,500]$ and $[0,360]$ respectively. The orientation is uniformly distributed in $\left[40^{\circ}, 140^{\circ}\right]$. (b) Result of $n=6$ thinning applied to part (a). (c) Polynomial fit of order $m=7$ to part (b) (solid line), compared with the actual surface (dashed line). $E_{1}=2.28 \mathrm{~cm}, E_{2}=0.096$. (d) The result of spatial voting and subsequent thresholding. (e) Polynomial fit of order $m=7$ to part (d) (solid line), and the actual surface (dashed line). $E_{1}=5.34 \mathrm{~cm}, E_{2}=0.224$ (simulated data).

of five ultrasonic sensors has been moved horizontally over a distance of $1.5 \mathrm{~m}$ to increase the total number of arcs, collecting data every $2.5 \mathrm{~cm}$. In the resulting arc map, there are some arcs which are not tangent to the actual surface at any point (e.g. the isolated arcs faintly visible in the upper part of figure 6(a)). These correspond to spurious data due to higher-order reflections, readings from other objects in the environment or totally erroneous readings. Such points are readily eliminated upon processing (figure 6(b) and (d)). The polynomial fit to part (b) is shown in figure 6(c), with $E_{1}=2.09 \mathrm{~cm}, E_{2}=0.204$. Corresponding results obtained with spatial voting and thresholding are given in parts (d) and (e). In this case, the errors are $E_{1}=2.12 \mathrm{~cm}, E_{2}=0.206$. For this experimental example, the actual surface profile was determined by using a very accurate (and much more expensive) laser structured-light system [21].

Although structured arrays such as linear or circular ones are often preferred in theoretical work for simplicity and ease of analysis, the method presented here can handle irregular arrays equally easily. In fact, the large number of simulations we have undertaken indicate that arrays consisting of irregularly located and oriented sensors tend to yield better results. This stems from the fact that the many different vantage points and orientations of the sensors tend to complement each other better than in the case of a structured array. Although the problem of optimal complementary sensor placement is a subject for future research, the large number of simulations performed indicate that it is preferable to work with irregular arrays rather than simple-structured arrays such as linear or circular ones. A detailed study of the effect of using structured sensor configurations such as linear and circular arrays, as well as irregularly configured and moving sensors in conjunction with the morphological processing approach can be found in [18]. Although the elements of the irregular transducer array may obscure each other under certain circumstances, such configurations may also correspond to a synthetic array (as described after the first example in this section). Even when it is the case that SAR techniques are not used, the perturbation on the fields caused by other transducers will be negligible if the transducers are relatively small. In those cases where the obstruction cannot be neglected, the only modification required in the algorithm is to simply omit those arcs corresponding to such transducers. The morphological processing approach has also been applied to map building for mobile robots using experimentally obtained ultrasonic data from test rooms involving target types commonly encountered in robot environments [22].

In the next example, the locations and the line-of-sight orientations of the sensors are generated randomly and do not conform to any special structure. Figure 7(a) illustrates an arc map obtained with such a sensor configuration. In figure 7(b), the result obtained after applying $n=2$ thinning to the arc map in part (a) are shown. Applying spatial voting and thresholding to the arc map in part (a), the result in part (d) is obtained. The curves fitted are presented in parts (c) and (e) of the same figure. Morphological processing followed by curve fitting results in $E_{1}=2.28 \mathrm{~cm}, E_{2}=0.096$. On the other hand, the errors obtained by spatial voting and 
Table 1. Results of various morphological operations on the sinusoidal surface.

\begin{tabular}{llll}
\hline Morphological operation & $E_{1}(\mathrm{~cm})$ & $E_{2}$ & $t_{\mathrm{CPU}}(\mathrm{s})$ \\
\hline thinning $(n=1:$ pruning) & 2.41 & 0.236 & 0.29 \\
thinning $(n=2)$ & 2.21 & 0.217 & 0.28 \\
thinning $(n=3)$ & 2.03 & 0.199 & 0.27 \\
thinning $(n=4)$ & 2.09 & 0.205 & 0.27 \\
thinning $(n=5)$ & 2.46 & 0.242 & 0.26 \\
\hline
\end{tabular}

subsequent thresholding and curve fitting are $E_{1}=5.34 \mathrm{~cm}$, $E_{2}=0.224$, which are larger by a factor of 2.34 .

Our final example deals with a surface whose profile is a sinusoid with amplitude $30 \mathrm{~cm}$, period $125 \mathrm{~cm}$, and which is located at a distance of $2.0 \mathrm{~m}$ from $y=0$. The arc map generated by using an array of 36 arbitrarily located and oriented sensors is given in figure 8(a). The result of $n=3$ thinning is given in part (b). The fitted curve and its comparison with the original surface are presented in part (c) of the same figure, resulting in $E_{1}=1.98 \mathrm{~cm}$, $E_{2}=0.195$. Corresponding results obtained with spatial voting and thresholding are given in parts (d) and (e), where $E_{1}=3.81 \mathrm{~cm}, E_{2}=0.376$. The errors obtained with the second method are greater than those obtained with morphological processing by a factor of 1.92 .

Overall, we observe that the errors obtained with morphological processing are lower than those obtained with spatial voting. In our examples, the errors obtained with the former approach have been found to be up to $80 \%$ lower than those obtained with the latter approach.

In the above examples, the values of $n$ and $m$ and the value of the threshold employed are those which yield the smallest error $E_{1}$ (and consequently $E_{2}$ ). For the examples given, threshold values between 2 and 6 were found to give the best results. The optimal value of the threshold depends on the amount of measurement noise, the resolution of the arc map and the total number of arcs, which further depends on the number and configuration of the sensors used. In the simulations, where the actual surface profile is known, it is possible to choose the optimal value of $n$ and $m$ or the threshold, minimizing $E_{1}$ or $E_{2}$. In practice, this is not possible so that one must use parameter values judged appropriate for the given system and the class of surfaces under investigation, based on previous simulations and experiments. This is indeed a feasible strategy because the precise choice among functionally similar morphological operations does not have a strong effect on the resulting errors, as exemplified by table 1 corresponding to figure 8 .

Although the algorithms were not actually run in real time, we have kept a record of the CPU times of the morphological operations (table 1). (Since the thresholding technique is much simpler than morphological processing algorithms, it would even take less time.) The total processing time consists of the data collection time plus the morphological processing time. Morphological operations are implemented in the $\mathrm{C}$ programming language and the programs are run on a $200 \mathrm{MHz}$ Pentium Pro PC. The average CPU times for morphological processing, starting with the raw TOF data, are in general fractions of a second, indicating that the method is viable for real-time applications. On the other hand, the time it takes for an array of 16 sonars to collect all the TOF data is $16 \times 40 \mathrm{~ms}=0.64 \mathrm{~s}$ which is of the same order of magnitude as the morphological processing time. It should be noted that the actual algorithmic processing time is a small fraction of the CPU time, as most of the CPU time is consumed by file operations, reads and writes to disk, matrix allocations etc. Thus, it seems possible that a dedicated system could extract the surface profile even faster, bringing the computation time much below the data collection time.

\subsection{Discussion}

Of the two methods described, morphological processing emphasizes neighbouring relationships whereas the spatial voting method takes into account the number of arcs which intersect at a certain pixel. From a different perspective, the morphological processing approach can also be interpreted as a kind of spatial voting scheme where the neighbours of a certain pixel provide local support and reinforcement by voting for it. In this perspective, the two approaches differ in the specific way the voting is realized.

From a map-building perspective, the methods can also be considered as hybrids of feature-based and grid-based methods. Initially, the environment is discretized into a rectangular array of square cells. After accumulation of a sufficient amount of measurements and their processing using either one of the methods, a curve-fitting procedure is employed to extract the geometry of the surface under investigation. The voting method essentially relies on the accumulation of multiple measurements in each pixel so that it does not allow the use of very small grid sizes. On the other hand, the morphological processing method does not exhibit such a limitation.

\subsection{Choice of grid size}

In this section, we discuss the choice of sampling resolution or pixel size. There are a couple of factors that determine the accuracy of TOF readings in a range measurement system. One of these factors is the operating wavelength of the measurement system. Other factors include effects such as the thermal noise in the receiving circuitry or the ambient acoustic noise. Given these, it is not meaningful to choose the pixel size much smaller than the resolving limit determined by these factors since it would increase the computational burden without resulting in a more accurate profile determination. Thus, the pixel size should be chosen comparable to the TOF measurement accuracy. Nevertheless, since the TOF accuracy may not be known beforehand, in the following, we have also examined the cases where the noise or uncertainty is smaller, as well as larger than one pixel. The pixel size used throughout this study is $1 \mathrm{~cm}$.

\subsection{The effect of measurement noise}

To investigate the robustness of the morphological processing method to noise, zero-mean white Gaussian noise has been added to the TOF measurements. The noise standard deviation $\left(\sigma_{n}\right)$ is varied logarithmically to cover a broad range of noise levels, as shown in figure 9. As expected, for $\sigma_{n}$ smaller than one pixel, the performance is approximately the 


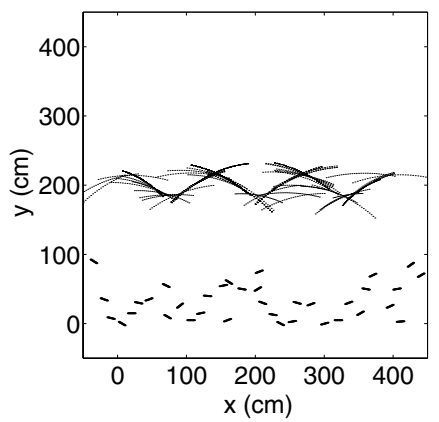

(a)

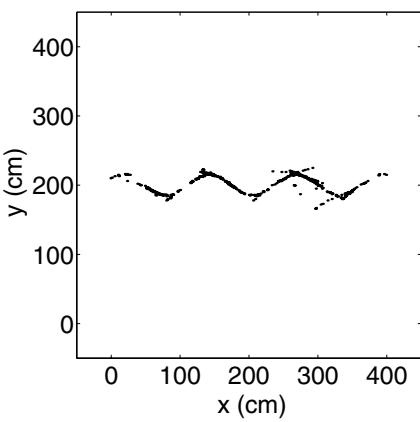

(b)

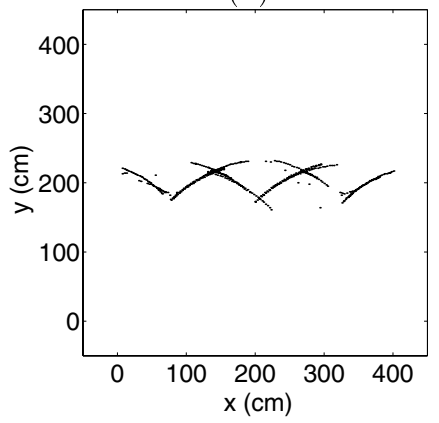

$(\mathrm{d})$

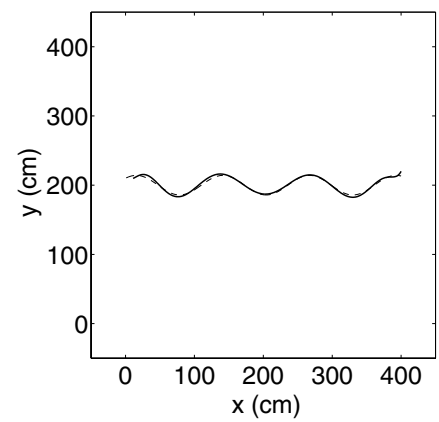

(c)

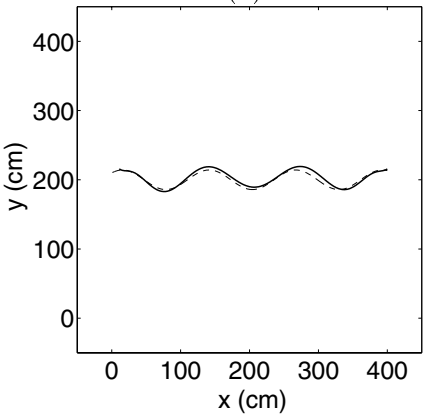

(e)

Figure 8. (a) The arc map of a sinusoidal surface obtained with an array of 36 sensors, each of $30^{\circ}$ beamwidth, (b) the result of $n=3$ thinning, (c) polynomial fit of order $m=10$ (solid line) and the original surface (dashed line). $E_{1}=1.98 \mathrm{~cm}, E_{2}=0.195$. (d) The result of spatial voting and subsequent thresholding, (e) polynomial fit of order $m=10$ to part (d) (solid line), and the actual surface (dashed line). $E_{1}=3.81 \mathrm{~cm}, E_{2}=0.376$ (simulated data).

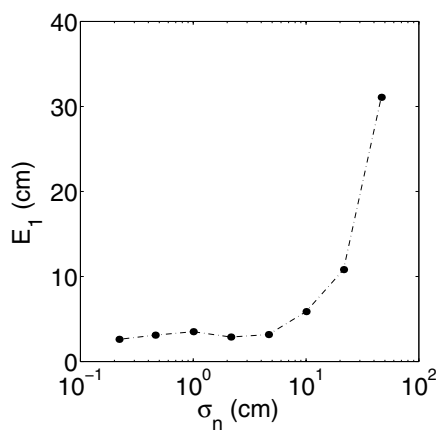

(a)

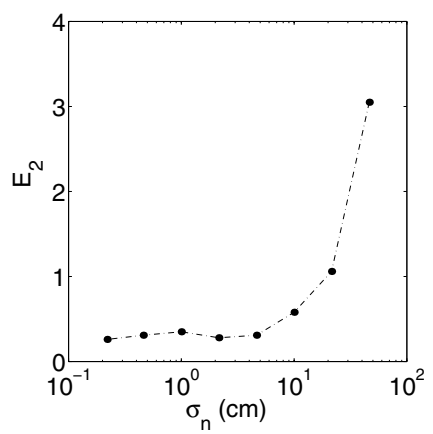

(b)

Figure 9. (a) $E_{1}$, (b) $E_{2}$, as the standard deviation of the noise $\sigma_{n}$ on the TOF readings is increased.

same as for the noiseless case. This performance can be further improved by reducing the pixel size until it becomes comparable to the TOF measurement accuracy, at the cost of greater computation time.

As expected, the error increases as the noise level increases beyond one pixel (figure 9). Since the method relies on the mutual reinforcement of several arcs to reveal the surface, larger amounts of noise are expected to have a destructive effect on this process by moving the various arc segments out of their mutually reinforcing positions. Despite what is suggested by these arguments, the error does not start increasing drastically until $\sigma_{n}=10 \mathrm{~cm}$, up to which the method seems to be reasonably robust to noise and the performance is comparable to the noiseless case. This is partly because the least-squares polynomial fit helps eliminate some of the noise.
Figure 10(a) illustrates the arc map obtained from the same surface as in figure 8 , except that zero-mean white Gaussian noise of standard deviation $5 \mathrm{~cm}$ has been added to the TOF measurements. The result of $n=3$ thinning is given in part (b). The polynomial fit and its comparison with the original surface are presented in part (c) of the same figure, yielding $E_{1}=4.62 \mathrm{~cm}, E_{2}=0.454$. Corresponding results obtained with spatial voting and thresholding are given in parts (d) and (e), with $E_{1}=5.38 \mathrm{~cm}, E_{2}=0.528$. The errors obtained with the second method are greater than those obtained with the first method by a factor of 1.16 .

\subsection{The effect of surface roughness}

Even though the two methods we have discussed were initially developed and demonstrated for specularly reflecting 


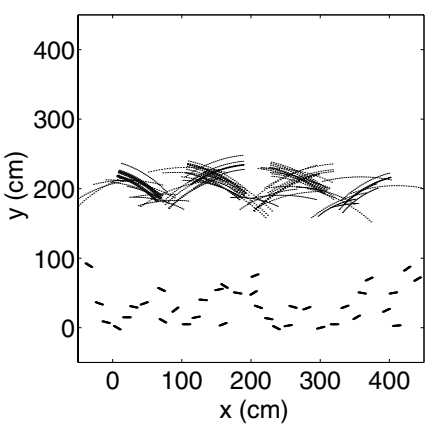

(a)

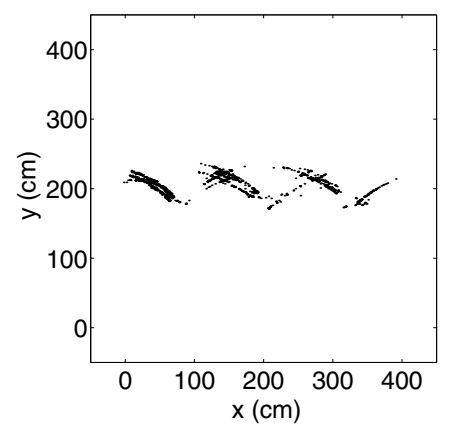

(b)

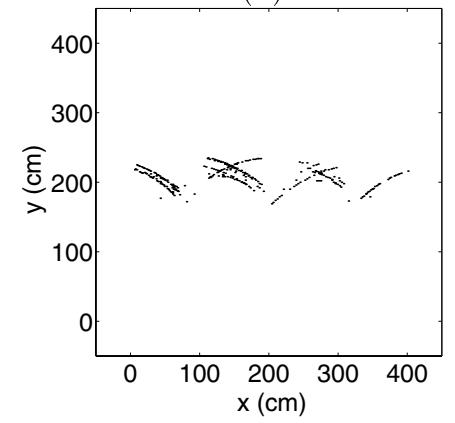

(d)

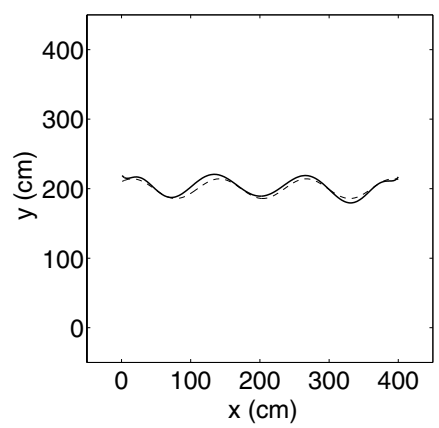

(c)

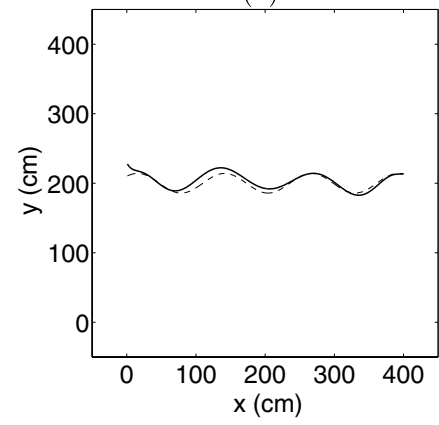

(e)

Figure 10. (a) The arc map of the sinusoidal surface obtained from noisy TOF measurements $\left(\sigma_{n}=5 \mathrm{~cm}\right)$, (b) the result of $n=3$ thinning, (c) polynomial fit of order $m=10$ to part (b) (solid line), and the original surface (dashed line), $E_{1}=4.62 \mathrm{~cm}, E_{2}=0.454$, (d) the result of spatial voting and subsequent thresholding, (e) polynomial fit of order $m=10$ to part (d) (solid line), and the actual surface (dashed line). $E_{1}=5.38 \mathrm{~cm}, E_{2}=0.528$ (simulated data).

surfaces, subsequent tests were performed with Lambertian surfaces of varying roughness. Arc maps obtained from rough surfaces generally resemble those from specularly reflecting surfaces, except that the arcs are now slightly more dispersed.

We now reconsider the example of figure 6 given in section 3.4. Figure 11 illustrates the morphological processing results obtained from this surface when its roughness is varied by covering it with two different types of material. The original surface in figure 6(a) was made of smooth, thin cardboard. The results in parts (a) and (b) of figure 11 were obtained when the same surface was covered with packing material with small and large air bubbles, respectively. The packing material with small bubbles had a honeycomb pattern of uniformly distributed circular bubbles of diameter $1.0 \mathrm{~cm}$ and height $0.3 \mathrm{~cm}$, with a centre-tocentre separation of $1.2 \mathrm{~cm}$. The packing material with large bubbles had the same pattern with diameter, height and centre-to-centre separation $2.5 \mathrm{~cm}, 1.0 \mathrm{~cm}$ and $2.8 \mathrm{~cm}$ respectively. The corresponding results obtained with spatial voting and subsequent thresholding are provided in figure 12. The results indicate that both methods still work for rough surfaces, with slightly larger errors. The errors obtained with the second method are still larger than those obtained with morphological processing. Table 2 provides a comparison of the errors obtained with the two methods. Overall, the errors for rough surfaces are larger than those in figure 6 . The morphological approach maintains its superiority with respect to spatial voting.
Table 2. Comparison of the two approaches on surfaces with different roughness.

\begin{tabular}{lll}
\hline Surface material & Method & $E_{1}(\mathrm{~cm})$ \\
\hline thin cardboard & morphological & \\
& processing & 2.09 \\
& spatial voting & 2.12 \\
small-bubbled & morphological & \\
packing material & processing & 3.56 \\
& spatial voting & 4.08 \\
large-bubbled & morphological & \\
packing material & processing & 3.97 \\
& spatial voting & 4.38 \\
\hline
\end{tabular}

\section{Conclusion}

Two novel methods are described for determining arbitrary surface profiles from data acquired by ultrasonic range sensors, by applying morphological processing and spatial voting followed by thresholding. Both methods are extremely flexible, versatile and robust, as well as being simple and straightforward. They can deal with arbitrary numbers and configurations of sensors, including synthetic arrays. Resolution improves with the number of sensors used. Accuracies down to a few centimetres were demonstrated in our experiments. The methods are robust in many aspects; they have the inherent ability to eliminate undesired TOF readings arising from higher-order reflections, crosstalk and noise, as well as processing multiple echoes informatively. Of the two methods, morphological processing has been found to be generally superior compared 

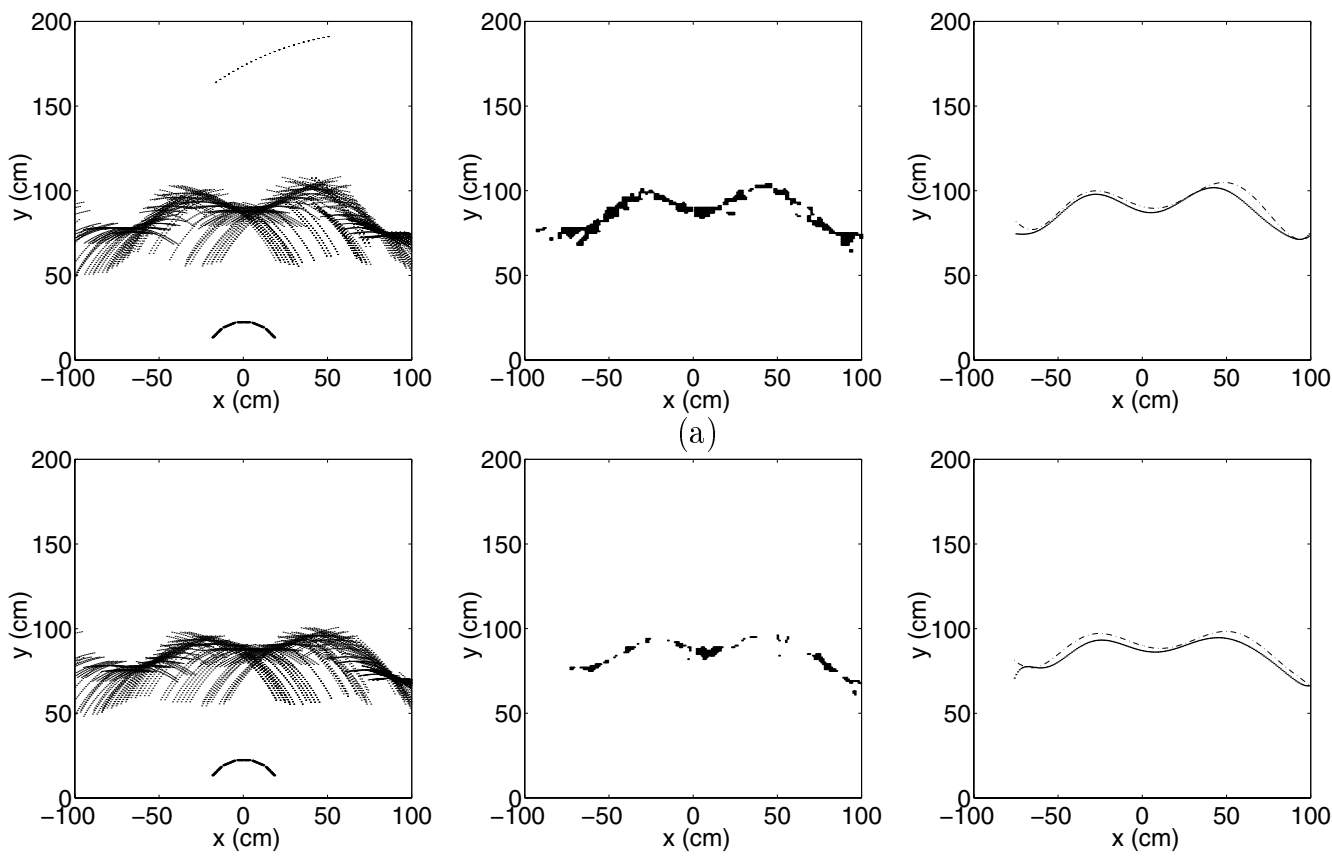

(b)

Figure 11. Morphological processing results from surfaces of the same shape but of increasing roughness: (a) packing material with small bubbles: $E_{1}=3.56 \mathrm{~cm}$, (b) packing material with large bubbles: $E_{1}=3.97 \mathrm{~cm}$ (experimental data).
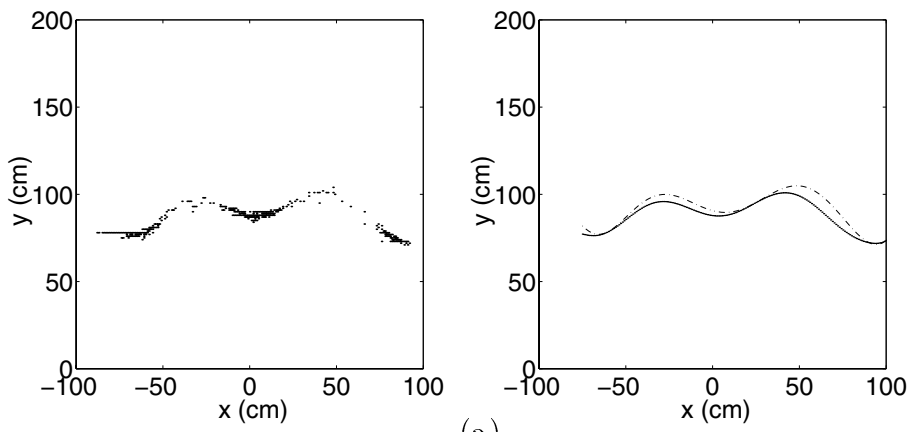

(a)
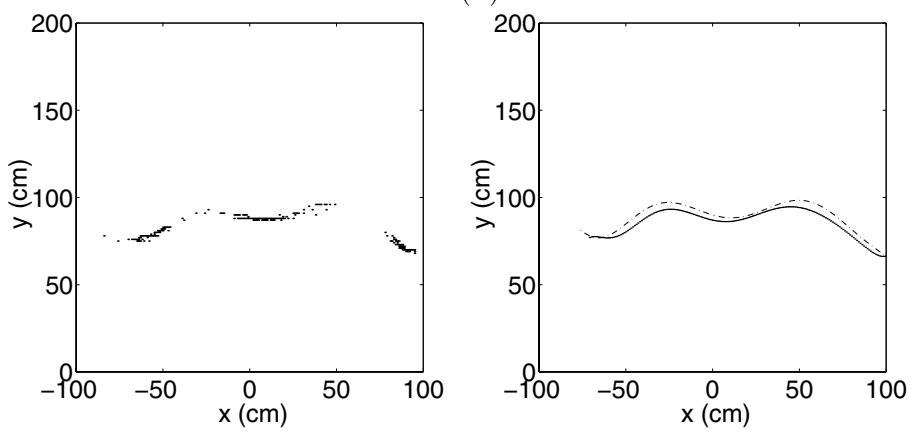

(b)

Figure 12. Spatial voting results from surfaces of the same shape but of increasing roughness: (a) packing material with small bubbles: $E_{1}=4.08 \mathrm{~cm}$, (b) packing material with large bubbles: $E_{1}=4.38 \mathrm{~cm}$ (experimental data).

to spatial voting followed by thresholding. It maintains its superiority when noisy measurements and rough surfaces are involved.

The CPU times for arc map processing (when implemented in the $\mathrm{C}$ programming language and run on a $200 \mathrm{MHz}$ Pentium Pro PC) are generally about a quarter of a second [22], indicating that the methods are viable for realtime applications.

To the best of our knowledge, this is the first application of morphological processing techniques to sonar range data. The method developed extends the types of object that can be handled by intelligent systems from simple primitives 
such as planes, corners, edges, cylinders, to also include those with arbitrarily curved surfaces. This is a substantial generalization, increasing the recognition and navigation capability of such systems. The methods presented in this paper, which are based on the use of multiple range sensors combined with sonar arc map processing, are very basic and general so that they can be applied to different physical modalities of range sensing of vastly different scales and in many different areas. These may include radar, sonar, optical sensing and metrology, remote sensing, ocean surface exploration, geophysical exploration, robotics and intelligent systems and acoustic microscopy. In our case, the application motivating our research was map building for mobile robotics. For instance, the system demonstrated can be used for continual real-time map building purposes on a robot navigating in a given environment. The robot can continually add to and update its collection of arcs and reprocess them as it moves, effectively resulting in a synthetic array with more sensors than the robot actually has. Apart from indoor mobile robotics, the methods can also find application in outdoor land vehicles and other intelligent systems operating underwater, underground or in outer space, and dealing with rough terrain or other curved surfaces.

Both methods can be readily generalized to threedimensional environments with the arcs replaced by spherical or elliptical caps and the arc map processing rules extended to three dimensions [3]. In certain problems, it may be preferable to reformulate the methods in polar or spherical coordinates. Some applications may involve an inhomogeneous and/or anisotropic medium of propagation. It is envisioned that the methods could be generalized in such cases by constructing broken or non-ellipsoidal arcs.

\section{Acknowledgment}

This work was partially supported by TÜBITAK under grants 197E051, EEEAG-116 and EEEAG-92.

\section{References}

[1] Barshan B and Kuc R 1990 IEEE Trans. Pattern Anal. Machine Intell. 12 560-9

[2] Brown M K 1986 Int. J. Robot. Res. 5 3-18

[3] Preston K 1991 Image Vision Comput. 9 285-95

[4] Hauptmann P 1993 Sensors: Principles and Applications (Englewood Cliffs, NJ: Prentice-Hall)

[5] Polaroid Corporation 1997 Polaroid Manual Ultrasonic Components Group, 119 Windsor Street, Cambridge, MA, USA

[6] Dougherty E R 1992 An Introduction to Morphological Image Processing (Bellingham, WA: SPIE)

[7] Yu P, Anastassopoulos V and Venetsanopoulos A N 1996 Math. Comput. Simul. 40 577-95

[8] Brivot R and Marchant J A 1996 IEE Proc. Vision Image Signal Processing 143 118-24

[9] Mojsilovic A, Popovic M, Amodaj N, Babic R and Ostojic M 1997 Ann. Biomed. Eng. 25 1059-71

[10] Krishnamurthy S, Iyengar S S, Holyer R J and Lybanon M 1994 IEEE Trans. Geosci. Remote Sensing 32 759-67

[11] Cardillo J and Sidahmed M A 1996 Pattern Recognition 29 $27-49$

[12] Saniie J and Mohamed M A 1994 IEEE Trans. Ultrason. Ferroelectron. Frequency Control 41 150-60

[13] Wilson D L, Dalal P, Kump K S, Benard W, Xue P, Marchant R E and Eppell S J 1996 J. Vac. Sci. Technol. B 14 2407-16

[14] Pitas I and Maglara A 1991 Pattern Recognition 24 165-81

[15] Verly J G and Delanoy R L 1993 Opt. Eng. 32 3295-306

[16] Pitas I 1993 Digital Image Processing Algorithms (Hemel Hempstead: Prentice-Hall)

[17] Myler H R and Weeks A R 1993 Computer Imaging Recipes in $C$ (Englewood Cliffs, NJ: Prentice-Hall)

[18] Başkent D 1998 Surface profile determination from multiple sonar data using morphological processing Master's Thesis Bilkent University, Department of Electrical Engineering, Ankara, Turkey

[19] Skolnik M I 1981 Introduction to Radar Systems (New York: McGraw-Hill)

[20] Nomadic Technologies, Inc. 1997 Nomad 200 Manual Mountain View, CA

[21] Nomadic Technologies, Inc. 1997 The Sensus 500 User's Guide Mountain View, CA

[22] Başkent D and Barshan B 1999 Int. J. Robot. Res. 18 788-808 